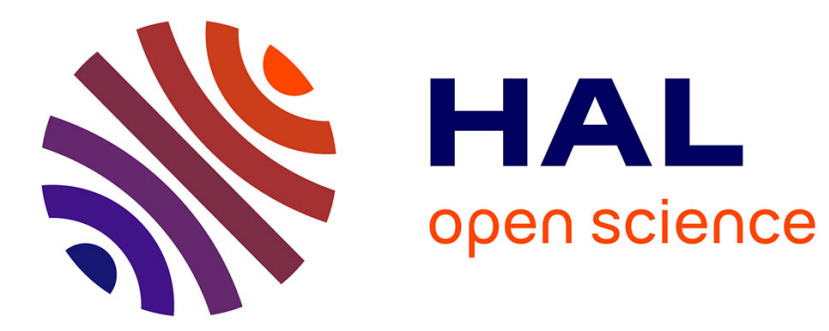

\title{
Computing the Fundamental Group in Digital Space
}

Rémy Malgouyres

\section{To cite this version:}

Rémy Malgouyres. Computing the Fundamental Group in Digital Space. International Journal on Pattern Recognition and Artificial Intelligence (IJPRAI), 2001, 15 (7), pp.1075-1088. 10.1142/S0218001401001325 . hal-01319678

\section{HAL Id: hal-01319678 \\ https://hal.science/hal-01319678}

Submitted on 22 May 2016

HAL is a multi-disciplinary open access archive for the deposit and dissemination of scientific research documents, whether they are published or not. The documents may come from teaching and research institutions in France or abroad, or from public or private research centers.
L'archive ouverte pluridisciplinaire HAL, est destinée au dépôt et à la diffusion de documents scientifiques de niveau recherche, publiés ou non, émanant des établissements d'enseignement et de recherche français ou étrangers, des laboratoires publics ou privés. 


\title{
Computing the Fundamental Group in Digital Spaces
}

\author{
Rémy Malgouyres \\ GREYC, ISMRA, 6, bd Mal Juin, F-14000 CAEN, FRANCE. \\ E-mail: Malgouyres@greyc.ismra.fr
}

\begin{abstract}
As its analogue in the continuous framework, the digital fundamental group represents a major information on the topology of discrete objects. However, the fundamental group is an abstract information and cannot directly be encoded in a computer using its definition. A classical mathematical way to encode a discrete group is to find a presentation of this group. In this paper, we construct a presentation for the fundamental group of an arbitrary graph, and a finite presentation (hence encodable in the memory of a computer) of any subset of $\mathbb{Z}^{3}$. This presentation can be computed by an efficient algorithm.
\end{abstract}

Key words: Pattern Recognition, Digital topology, graphs, Homotopy.

\section{Introduction}

As its analogue in the continuous framework, the digital fundamental group, originally introduced by T. Y. Kong in [4] in the 3D case (see also [5]), represents a major information on the topology of discrete objects. It is in particular related to the notion of a simple point in 3D ([2], see also [1]), and an even closer relationship between the fundamental group and topology preservation has been established within digital surfaces ([10] and [3]).

For these reasons, we would obtain a very powerfull tool for pattern recognition if we could make the information of the fundamental group accessible to computers in the 3D case. An even more difficult problem is to find an algorithm to determine whether two given 3D discrete objects have isomorphic fundamental groups. Such an algorithm would represent a significant step to determine whether two objects could be the same up to some "continuous deformation".

The fundamental group contains strictly more information than the first homology group. However, whereas the first homology group is always accessible to computers, it is somehow difficult to transform the abstract notion of the fundamental group into some data which can be handled by computers. A classical way to encode certain types of discrete groups is to find presentations of these groups. A presentation of a group is a model of the group up to isomorphism, which is characterized by an integer and some words on an alphabet called relators. Such a data can easily be encoded in a computer. 
In [8], the author studies the planar 2D case. In this case, the fundamental group is shown to be isomorphic to a free group (i.e. there are no relators in this case). This is an ideal case since computations in the free groups are easy to perform, and the type of the group up to isomorphism is easily determined. In [9], the case of digital surfaces is treated. In this paper, we introduce the digital fundamental group in a very general fromework of graph, and we construct a presentation of the fundamental group in this field. Then we solve the general 3D case, for which there are interesting potential applications to pattern recognition, by constructively providing a finite presentation of fundamental groups of 3D digital object.

The paper is organized as follows. First we recall some basic algebraic preliminaries about groups, quotient groups, free groups, and groups given by generators and relations. Afterwards, we define the fundamental group for an arbitrary graph, with respect to an arbitrary set of null loops, and we construct a presentation for this group. Then we set the required definitions of 3D digital topology (adjacencies, paths, connectivity, and the digital fundamental group), and we show how a presentation of the fundamental group of any connected subset of $\mathbb{Z}^{3}$ can be obtained as a particular case of a graph fundamental group. The word connected for a subset of $\mathbb{Z}^{3}$ can be understood according to any of the couples of adjacency relations $(26,6),(18,6)$, $(6,26),(6,18)$, Finaly we explain how to reduce the number of generators and relators of the obtained presentation. Our proof is constructive and leads to an efficient algorithm to compute a presentation of 3D fundamental groups.

\section{Basic Definitions and Notations}

\subsection{Groups, Normal Subgroups}

We consider a group $(\Gamma, *)$ with $1_{\Gamma}$ as unit element. Let $h \in \Gamma$. A conjugate of $h$ in $\Gamma$ is an element of $\Gamma$ of the form: $g * h * g^{-1}$ with $g \in \Gamma$. Now let $H$ be a subgroup of $\Gamma$, we say that $H$ is normal in $\Gamma$ if for any $h \in H$, any conjugate of $h$ in $\Gamma$ belongs to $H$.

Given $H$ a normal subgroup of $\Gamma$, we consider $\equiv_{H}$ the relation on $\Gamma$ defined by $\left[g \equiv_{H}\right.$ $\left.g^{\prime}\right] \Longleftrightarrow\left[g^{\prime} * g^{-1} \in H\right]$. Since $H$ is a normal subgroup of $\Gamma$, this is an equivalence relation. We denote by $\Gamma / H$ the set of equivalence classes of elements of $\Gamma$ under the relation $\equiv_{H}$, and by $p_{H}: \Gamma \longrightarrow \Gamma / H$ the projection which to an element of $\Gamma$ associates its equivalence class under $\equiv_{H}$. Now, if $g \equiv_{H} g^{\prime}$ and $g_{1} \equiv_{H} g_{1}^{\prime}$, then since $H$ is normal we have $g * g_{1} \equiv_{H} g^{\prime} * g_{1}^{\prime}$. Hence the product $*$ defines an operation, which we also denote by $*$, on $\Gamma / H$. To $p_{H}(g)$ and $p_{H}\left(g^{\prime}\right)$, this operation associates $p_{H}\left(g * g^{\prime}\right)$. Obviously, the element $p_{H}\left(1_{\Gamma}\right)$ of $\Gamma / H$ is a unit element for the operation $*$ on $\Gamma / H$. Furthermore, given $g \in \Gamma$, the element $p_{H}\left(g^{-1}\right)$ of $\Gamma / H$ is an inverse for $p_{H}(g)$. Therefore, $(\Gamma / H, *)$ is a group which we call quotient group of $\Gamma$ by the normal subgroup $H$. Intuitively, all elements of $H$ are collapsed with the unit element to obtain the quotient group.

Now let $P$ be any subset of $\Gamma$. We consider the subset $H$ of $\Gamma$ composed of all products of conjugates of elements of $P$ and inverses of elements of $P$. Then $H$ is a normal subgroup of $\Gamma$ which we call normal subgroup of $\Gamma$ generated by $P$. The normal subgroup generated by $P$ is the smallest normal subgroup of $\Gamma$ which contains $P$. 


\subsection{Free Group, Generators and Relations}

Now, before to introduce groups given by generators and relations, we must introduce the notion of the (non abelian) free group with $m$ generators. Let $\left\{a_{1}, \ldots, a_{m}\right\} \cup\left\{a_{1}^{-1}, \ldots, a_{m}^{-1}\right\}$ be an alphabet with $2 m$ distinct letters, and let $\mathcal{W}_{m}$ be the set of the all words over this alphabet (i.e. finite sequences of letters of the alphabet). We say that two words $w \in \mathcal{W}_{m}$ and $w^{\prime} \in \mathcal{W}_{m}$ are the same up to an elementary simplification if, either $w$ can be obtained from $w^{\prime}$ by inserting in $w^{\prime}$ a sequence of the form $a_{i} a_{i}^{-1}$ or a sequence of the form $a_{i}^{-1} a_{i}$ with $i \in\{1, \ldots, m\}$, or $w^{\prime}$ can be obtained from $w$ by inserting in $w$ a sequence of the form $a_{i} a_{i}^{-1}$ or a sequence of the form $a_{i}^{-1} a_{i}$ with $i \in\{1, \ldots, m\}$. Now, two words $w \in \mathcal{W}_{m}$ and $w^{\prime} \in \mathcal{W}_{m}$ are said to be free equivalent if there is a finite sequence $w=w_{1}, \ldots, w_{k}=w^{\prime}$ of words of $\mathcal{W}_{m}$ such that for $i=2, \ldots, k$ the word $w_{i-1}$ and $w_{i}$ are the same up to an elementary simplification. This defines an equivalence relation on $\mathcal{W}_{m}$, and we denote by $\mathcal{F}_{m}$ the set of equivalence classes of this equivalence relation. If $w \in \mathcal{W}_{m}$, we denote by $\bar{w}$ the class of $w$ under the free equivalence relation. The concatenation of words defines an operation on $\mathcal{F}_{m}$ which provides $\mathcal{F}_{m}$ with a group structure. The group thus defined is called the free group with $m$ generators.

We denote by $1_{m}$ the unit element of $\mathcal{F}_{m}$, which is equal to $\bar{w}$ where $w$ is the empty word. The only result which we shall admit on the free group is the classical result that if a word $w \in \mathcal{W}_{m}$ is such that $\bar{w}=1_{m}$ and $w$ is not the empty word, then there exists in $w$ two successive letters $a_{i} a_{i}^{-1}$ or $a_{i}^{-1} a_{i}$ with $i \in\{1, \ldots, m\}$. This remark leads to an immediate algorithm to decide whether a word $w \in \mathcal{W}_{m}$ is such that $\bar{w}=1_{m}$. If $w=a_{i_{1}}^{\varepsilon_{1}} \cdots a_{i_{p}}^{\varepsilon_{p}}$ is a word of $\mathcal{W}_{m}$, we denote by $w^{-1}$ the word $w^{-1}=a_{i_{p}}^{-\varepsilon_{p}} \cdots a_{i_{1}}^{-\varepsilon_{1}}$. We have: $(\bar{w})^{-1}=\overline{w^{-1}}$.

Now we introduce the groups given by generators and relations. We consider $m \in \mathbb{N}^{*}$ and a finite subset $\mathcal{R}$ of $\mathcal{W}_{m}$. In this context, we call elements of $\mathcal{R}$ relators. We want to define a group from the free group with $m$ generators, in which the words of $\mathcal{R}$ represent the unit element. This group, called the group with $m$ generators and the relations of $\mathcal{R}$, is the quotient of the free group $\mathcal{F}_{m}$ by the normal subgroup generated by the set of the equivalence classes in $\mathcal{F}_{m}$ of elements of $\mathcal{R}$. Now, given $\Pi$ a group, finding a presentation of $\Pi$ is finding an (explicit) isomorphism from $\Pi$ to a group given by generators and relations.

\subsection{Fundamental Groups in Graphs}

Let $G=(V, E)$ be an antireflexive undirected graph, where $V$ is a set of vertices and $E$ is a set of pairs $\{x, y\}$, with $x \in V$ and $y \in V$, called edges. Two vertices $x, y \in V$ of $G$ are called adjacent in $G$ if $\{x, y\} \in E$.

A path $c$ in the graph $G$ is a sequence $c=\left(x_{0}, \ldots, x_{p}\right)$ of vertices of $G$ such that for $i=1, \ldots, p$ the vertex $x_{i}$ is adjacent in $G$ to the vertex $x_{i-1}$. The vertex $x_{0}$ is called the origin of $c$, and the vertex $x_{p}$ is called the end of $c$. A path $c$ in $G$ is called a loop if its origin is equal to its end.

Given two paths $c$ and $c^{\prime}$ in $G$ such that the end of $c$ is adjacent or equal to the origin of $c^{\prime}$, we denote by $c * c^{\prime}$ the concatenation of $c$ and $c^{\prime}$, which is also a path of $G$. Given $c=\left(x_{0}, \ldots, x_{p}\right)$ a path in $G$, we denote by $c^{-1}$ the reversed path $c^{-1}=\left(x_{p}, \ldots, x_{0}\right)$. A path of the form $c * c^{-1}$, where $c$ is a path of $G$, is called a back and forth in $G$.

Now we can define fundamental groups in $G$. Let $\mathcal{L}$ be any set of loops of $G$ containing all 
back and forths of $G$. The elements of $\mathcal{L}$ are called null loops. Given $c$ and $c^{\prime}$ two paths of $G$, we say that $c$ and $c^{\prime}$ are the same up to an elementary $\mathcal{L}$-deformation if $c$ and $c^{\prime}$ are of the form $c=\pi_{1} * \gamma * \pi_{2}$ and $c^{\prime}=\pi_{1} * \gamma^{\prime} * \pi_{2}$, where $\gamma * \gamma^{\prime-1}$ belongs to $\mathcal{L}$.

Two paths $c$ and $c^{\prime}$ in $G$ are called $\mathcal{L}$-homotopic in $G$ if there exists a sequence $\alpha_{0}, \ldots, \alpha_{q}$ of paths of $G$ such that $\alpha_{0}=c, \alpha_{q}=c^{\prime}$, and for $i=1, \ldots, q$ the paths $\alpha_{i}$ and $\alpha_{i-1}$ are the same up to an elementary $\mathcal{L}$-deformation.

Let $B$ be a fixed vertex in $G$, called the base vertex. We denote by $A_{B}(G)$ the set of all loops having $B$ as origin and end vertices. The $\mathcal{L}$-homotopy relation is an equivalence relation on $A_{B}(G)$, and we denote by $\Pi_{1}^{\mathcal{L}}(G, B)$ the set of the equivalence classes of this relation. Given $c \in A_{B}(G)$, we denote by $[c]$ the equivalence class of $c$ under the $\mathcal{L}$-homotopy equivalence relation, which is an element of $\Pi_{1}^{\mathcal{L}}(G, B)$.

The concatenation of loops is compatible with the $\mathcal{L}$-homotopy relation: if $[\alpha]=\left[\alpha^{\prime}\right]$ and $[\beta]=\left[\beta^{\prime}\right]$, then we must have $[\alpha * \beta]=\left[\alpha^{\prime} * \beta^{\prime}\right]$. Due to this property, the concatenation of loops defines an operation on $\Pi_{1}^{\mathcal{L}}(G, B)$, and this operation provides $\Pi_{1}^{\mathcal{L}}(G, B)$ with a group structure. We call this group the $\mathcal{L}$-fundamental group of $G$. Note that $\Pi_{1}^{\mathcal{L}}(G, B)$ is isomorphic (as a group) to $\Pi_{1}^{\mathcal{L}}\left(G, B^{\prime}\right)$ provided that $B$ and $B^{\prime}$ lie in the same connected component of $G$. The graph $G$ is called $\mathcal{L}$-simply connected if any path in $G$ is $\mathcal{L}$-homotopic to a trivial path reduced to a single vertex, or equivalently if $\Pi_{1}^{\mathcal{L}}(G, B)$ is reduced to a single element.

\section{Presentation of Fundamental Groups in Graphs}

In the sequel of this section, $G=(V, E)$ is a undirected antireflexive graph, and $\mathcal{L}$ is a set of loops in $G$ containing all back and forths of $G$, and elements of $\mathcal{L}$ are called null loops. Our purpose is to construct a presentation of the $\mathcal{L}$-fundamental group of $G$.

Let $G^{\prime}=\left(V, E^{\prime}\right)$, with $E^{\prime} \subset E$ be a spanning subgraph of $G$ which is connected and $\mathcal{L}^{\prime}$-simply connected, where $\mathcal{L}^{\prime}$ is the set of all elements of $\mathcal{L}$ which are paths of $G^{\prime}$. Such a subgraph $G^{\prime}$ can always be constructed, for instance by considering $G^{\prime}$ a covering tree of $G$.

Let us chose an arbitrary orientation on each edge of $G$ which is not an edge of $G^{\prime}$ : We consider $A=\left\{\left(l_{1}, r_{1}\right), \ldots,\left(l_{m}, r_{m}\right)\right\}$, where $E \backslash E^{\prime}=\left\{\left\{l_{1}, r_{1}\right\}, \ldots,\left\{l_{m}, r_{m}\right\}\right\}$ and $E \backslash E^{\prime}$ has cardinality $m$.

Let $\left\{a_{1}, \ldots, a_{m}, a_{1}^{-1}, \ldots, a_{m}^{-1}\right\}$ be an alphabet as considered in the definition of the free group, let $\mathcal{W}_{m}$ be the set of all words on this alphabet, and let $F_{m}$ be the free group with the $m$ generators $a_{1}, \ldots, a_{m}$.

First we associate a word $w(c)$ of $\mathcal{W}_{m}$ to each path $c$ in $G$. Let $c=\left(x_{0}, \ldots, x_{p}\right)$ be a path of $G$.

Definition 1 Let $i \in\{1, \ldots, m\}$ and $k \in\{0, \ldots, p-1\}$ be such that $\left\{l_{i}, r_{i}\right\}=\left\{x_{k}, x_{k+1}\right\}$. The couple $(k, i)$ is called an intersection of $c$ and $A$. If $\left(r_{i}, l_{i}\right)=\left(x_{k}, x_{k+1}\right)$, we say that the intersection $(k, i)$ is positive. If $\left(l_{i}, r_{i}\right)=\left(x_{k}, x_{k+1}\right)$, we say that the intersection $(k, i)$ is negative.

Notation 1 Given $(k, i)$ an intersection of $c$ with $A$, we denote by $\mathcal{O}_{c}(k, i)$ the number equal to +1 if the intersection $(k, i)$ is positive, and to -1 if the intersection $(k, i)$ is negative. 
Now, we define $w(c)$ as the word, containing one symbol $a_{i}^{\mathcal{O}_{c}(k, i)}$ for each intersection $(k, i)$ of $c$ and $A$, these symbols appearing in the word $w(c)$ in the order of the increasing $k$ (i.e. the order in which the intersections appear along $c$ ). We denote by $\overline{w(c)}$ the class of $w(c)$ up to elementary simplifications (see the definition of the free group). For the sake of simplicity, we shall often write $w(c)$ instead of $\overline{w(c)}$.

In order to define a group with generators and relations, let $\mathcal{R}=\{w(c) / c \in \mathcal{L}\}$ we the set of all words associated to loops of $\mathcal{L}$. The set $\mathcal{R}$ will be the set of our relators.

Let $H$ be the normal subgroup of $\mathcal{F}_{m}$ generated by all the words of $\mathcal{R}$. Finally, let $\Gamma=\mathcal{F}_{m} / H$ be the group with $m$ generators and the relations of $\mathcal{R}$. We shall prove that the $n$-fundamental group $\Pi_{1}^{\mathcal{L}}(G, B)$ of $G$ is isomorphic to the group $\Gamma$, which provides a presentation of $\Pi_{1}^{\mathcal{L}}(G, B)$.

We denote by $\equiv_{H}$ the relation of equality modulo $H$ in $\mathcal{F}_{m}$, and by $p_{H}: \mathcal{F}_{m} \longrightarrow \Gamma$ the projection (see the definition of the quotient group). Given $c$ a path of $A_{B}(G)$, we denote by $\varphi(c)$ the element $p_{H}(\overline{w(c)})$ of $\Gamma$.

Theorem 1 If $c$ and $c^{\prime}$ are two $\mathcal{L}$-homotopic paths of $G$, then $\varphi(c)=\varphi\left(c^{\prime}\right)$. In other words, the $\operatorname{map} \varphi: A_{B}(G) \longrightarrow \Gamma$ induces a map

$$
\left\{\begin{array}{ccc}
\widetilde{\varphi}: \Pi_{1}^{\mathcal{L}}(G, B) & \longrightarrow & \Gamma \\
{[c]} & \longmapsto & \widetilde{\varphi}([c])=\varphi(c)
\end{array}\right.
$$

Moreover, the map $\widetilde{\varphi}$ is clearly a group morphism.

Proof: It is sufficient to prove Theorem 1 in the case when $c$ and $c^{\prime}$ are the same up to an elementary $\mathcal{L}$-deformation. So, suppose $c$ and $c^{\prime}$ are of the form $c=\pi_{1} * \gamma * \pi_{2}$ and $c^{\prime}=\pi_{1} * \gamma^{\prime} * \pi_{2}$.

Since $\gamma * \gamma^{\prime-1} \in \mathcal{L}$, the word $w\left(\gamma * \gamma^{\prime-1}\right)$ belongs to $\mathcal{R}$ (from the very definition of $\mathcal{R}$ ), so that

$$
\begin{aligned}
\overline{w(c)} & =\overline{w\left(\pi_{1}\right)} * \overline{w(\gamma)} * \overline{w\left(\pi_{2}\right)} \\
& =\overline{w\left(\pi_{1}\right)} * \overline{w\left(\gamma * \gamma^{\prime-1}\right)} * \overline{w\left(\gamma^{\prime}\right)} * \overline{w\left(\pi_{2}\right)} \\
& \equiv{ }_{H} \\
& =\overline{w\left(\pi_{1}\right)} * \overline{w\left(\gamma^{\prime}\right)} * \overline{w\left(\pi_{2}\right)}
\end{aligned}
$$

Finally, $\overline{w(c)} \equiv_{H} \overline{w\left(c^{\prime}\right)}$ so that $\varphi(c)=\varphi\left(c^{\prime}\right)$.

The following result is the main result of this section :

Theorem 2 The map $\widetilde{\varphi}: \Pi_{1}^{\mathcal{L}}(G, B) \longrightarrow \Gamma$ is a group isomorphism.

In order to prove Theorem 2, we should first show two lemmas.

Lemma 1 Let $c$ be a path in $G$ and let $w \in \mathcal{W}_{m}$ be a word such that $\overline{w(c)}=\bar{w}$ in the free group $\mathcal{F}_{m}$. Then $c$ is $\mathcal{L}$-homotopic in $G$ to a path $c^{\prime}$ such that $w\left(c^{\prime}\right)=w$.

Proof of Lemma 1: It is sufficient to prove the result when $w(c)$ and $w$ are the same up to an elementary simplification. We distinguish two cases: 
First case: If $w$ is obtained by inserting in the word $w(c)$ a sequence $a_{i} a_{i}^{-1}$ or $a_{i}^{-1} a_{i}$ (say $\left.a_{i} a_{i}^{-1}\right)$. We decompose $w(c)=w_{1} w_{2}$ and $w=w_{1} a_{i} a_{i}^{-1} w_{2}$. We also decompose $c=c_{1} * c_{2}$ with $w\left(c_{1}\right)=w_{1}$ and $w\left(c_{2}\right)=w_{2}$. Let $\alpha$ be a path in the connected and simply connected graph $G^{\prime}$ from the last vertex of $c_{1}$ to the vertex $r_{i}$. Then the path $c^{\prime}=c_{1} * \alpha *\left(r_{i}, l_{i}, r_{i}\right) * \alpha^{-1} * c_{2}$ is clearly $\mathcal{L}$-homotopic in $G$ to $c=c_{1} * c_{2}$, and we have $w\left(c^{\prime}\right)=w_{1} * a_{i} a_{i}^{-1} w_{2}=w$.

Second case: If $w$ is obtained by deleting in $w(c)$ a subword of the form $a_{i} a_{i}^{-1}$ or $a_{i}^{-1} a_{i}$ (say $\left.a_{i} a_{i}^{-1}\right)$. We denote $w(c)=w_{1} a_{i} a_{i}^{-1} w_{2}$ and $w=w_{1} w_{2}$. We decompose $c=c_{1} * c_{2} * c_{3} * c_{4}$ with $w\left(c_{1}\right)=w_{1}, w\left(c_{2}\right)=a_{i}, w\left(c_{3}\right)=a_{i}^{-1}$ and $w\left(c_{4}\right)=w_{2}$.

Let $\left(k_{2}, i\right)$ be the unique intersection of $c_{2}$ with $A$, and let $\left(k_{3}, i\right)$ be the unique intersection of $c_{3}$ with $A$.

We denote $c_{2}=\alpha_{2} * \beta_{2}$ and $c_{3}=\alpha_{3} * \beta_{3}$, the last point of $\alpha_{2}$ and the first point of $\beta_{3}$ being equal to $r_{i}$, and the first point of $\beta_{2}$ and the last point of $\alpha_{3}$ being equal to $l_{i}$.

Since $G^{\prime}$ is simply connected, the closed path $\beta_{2} * \alpha_{3}$ in $G^{\prime}$ is $\mathcal{L}^{\prime}$-homotopic in $G^{\prime}$ (hence $\mathcal{L}$-homotopic in $G$ ) to the trivial path reduced to $\left(l_{i}, l_{i}\right)$. Hence $c$, which is equal to $c_{1} * c_{2} * c_{3} * c_{4}$, is equal to to $c_{1} * \alpha_{2} * \beta_{2} * \alpha_{3} * \beta_{3} * c_{4}$, so that it is $\mathcal{L}$-homotopic in $G$ to the path $c^{\prime}=c_{1} * \alpha_{2} * \beta_{3} * c_{4}$. Moreover, since $\alpha_{2} * \beta_{3}$ is a path of $G^{\prime}$, we have $w\left(c^{\prime}\right)=w\left(c_{1}\right) w\left(c_{4}\right)=w_{1} w_{2}=w$.

Lemma 2 Let $c$ be a path in $G$ such that $w(c)=w(\alpha)$ for $\alpha$ a null loop (i.e. $\alpha \in \mathcal{L})$. Then $c$ is $\mathcal{L}$-homotopic in $G$ to a path $c^{\prime}$ with $w\left(c^{\prime}\right)$ equal to the empty word (in other words, $c^{\prime}$ has no intersection with $A$ ).

Proof: We denote $w(\alpha)=a_{i_{1}}^{\varepsilon_{1}} \cdots a_{i_{p}}^{\varepsilon_{p}}$ with $i_{u} \in\{1, \ldots, m\}$ and $\varepsilon_{u} \in\{-1,+1\}$ for $u \in$ $\{1, \ldots, p\}$. For $u \in\{1, \ldots, p\}$, we denote by $\left(k_{u}, i_{u}\right)$ the $u^{\text {th }}$ intersection of $c$ with $A$, corresponding to the symbol $a_{i_{u}}^{\varepsilon_{u}}$ in $w(c)=w(\alpha)$. Finally, we decompose $c=c_{0} * \cdots * c_{p}$, where $c_{0}, \ldots, c_{p}$ are paths of the graph $G^{\prime}$, and we denote $y_{u}$ and $z_{u}$ respectively the first point and the last point of $c_{u}$ for $u \in\{0, \ldots, p\}$. Necessarily, for $u \in\{1, \ldots, p\}$, we have: $\left\{z_{u-1}, y_{u}\right\}=\left\{r_{i_{u}}, l_{i_{u}}\right\}$.

Since $G^{\prime}$ is simply connected, for $u=1, \ldots, p-1$, the path $c_{u}$ of $G^{\prime}$ is $\mathcal{L}^{\prime}$-homotopic in $G^{\prime}$ to a path $c_{u}^{\prime}$ of $G^{\prime}$ which is the portion of the path $\alpha$ between $y_{u}$ and $z_{u}$. The path $c_{1} * \cdots * c_{p-1}$ is $\mathcal{L}$-homotopic in $G$ to the path $c_{1}^{\prime} * \cdots * c_{p-1}^{\prime}$, which is $\mathcal{L}^{\prime}$-homotopic to a path $C$ of $G^{\prime}$ from $y_{1}$ to $z_{p-1}$ (namely, $C$ is the portion of $\alpha^{-1}$ between the first vertex of $c_{1}$ to the last vertex of $c_{p-1}$.

Hence, $c$ is $\mathcal{L}$-homotopic in $G$ to the path $c^{\prime}=c_{0} * C * c_{p}$, which is a path of $G^{\prime}$, so that $w\left(c^{\prime}\right)$ is the empty word.

Proof of Theorem 2: First we prove that the morphism $\widetilde{\varphi}$ is onto. To do this, it is sufficient to prove that for $i=1, \ldots, m$, there exists a loop $c$ of $A_{B}(G)$ such that $w(c)=a_{i}$. Let us consider $\left(l_{i}, r_{i}\right) \in A$. Since the graph $G^{\prime}$ is connected, there exists a path $c_{1}$ in $G^{\prime}$ from $B$ to $r_{i}$. Similarly, there exists a path $c_{2}$ in $G^{\prime}$ from $l_{i}$ to $B$. Now, the concatenation $c=c_{1} * c_{2}$ is a path in $G$ and we have $w(c)=a_{i}$. Hence, the morphism $\widetilde{\varphi}$ is onto.

There remains to prove that the morphism $\widetilde{\varphi}$ is one to one. To do this, we consider $c$ a loop of $A_{B}(G)$ such that $\widetilde{\varphi}([c])=1$ in $\Gamma$, and we have to prove that $c$ is $\mathcal{L}$-homotopic in $G$ to the trivial path $(B, B)$. 
We decompose $c=c_{1} * \cdots * c_{f}$, where for $k=1, \ldots, f$ the path $c_{k}$ is a path in the graph $G^{\prime}$, and for $k=1, \ldots, p-1$, the edge of $G$ between the last surfel of $c_{k}$ and the first surfel of $c_{k+1}$ is an edge of the form $\left\{r_{i}, l_{i}\right\}$.

Our hypothesis is that $\overline{w(c)}$ is in the normal subgroup $H$ of $\mathcal{F}_{m}$ generated by the elements of $\mathcal{R}$. Therefore, the word $w(c)$ is free equivalent to a word

$$
w=\prod_{a=1}^{g} w_{a} *\left(w\left(\alpha_{a}\right)\right)^{\varepsilon_{a}} * w_{a}^{-1}
$$

with for $a=1, \ldots g, w_{a} \in \mathcal{W}_{m}, \varepsilon_{a} \in\{-1,1\}$, and $\alpha_{a}$ is a loop of $\mathcal{L}$, From Lemma 1 , the path $c$ is $\mathcal{L}$-homotopic in $G$ to a path $c^{\prime}$ with $w\left(c^{\prime}\right)=w$. Let us decompose $c^{\prime}$ by setting $c^{\prime}=c_{1} * c_{2} * c_{3} * c_{4}$, with $w\left(c_{1}\right)=\prod_{a=1}^{g-1} w_{a} *\left(w\left(\alpha_{a}\right)\right)^{\varepsilon_{a}} * w_{a}^{-1}, w\left(c_{2}\right)=w_{g}, w\left(c_{3}\right)=\left(w\left(\alpha_{g}\right)\right)^{\varepsilon_{g}}$ and $w\left(c_{4}\right)=w_{g}^{-1}$.

From Lemma 2 follows that $c_{3}$ is $\mathcal{L}$-homotopic in $G$ with fixed extremities to a path $c_{3}^{\prime}$ which has no intersection with $A$, so that $w\left(c_{3}^{\prime}\right)$ is the empty word.

Now, $c^{\prime}$ is $\mathcal{L}$-homotopic in $G$ to the path $c^{\prime \prime}=c_{1} * c_{2} * c_{3}^{\prime} * c_{4}$, and $w\left(c^{\prime \prime}\right)=w\left(c_{1}\right) w_{g} w_{g}^{-1}$. Due to Lemma 1 , the path $c^{\prime \prime}$ is $\mathcal{L}$-homotopic to a path $c^{\prime \prime \prime}$ with

$$
w\left(c^{\prime \prime \prime}\right)=w\left(c_{1}\right)=\prod_{a=1}^{g-1} w_{a} *\left(R\left(i_{a}, s_{a}\right)\right)^{\varepsilon_{a}} * w_{a}^{-1} .
$$

It follows by induction that $c$ is $\mathcal{L}$-homotopic in $G$ to a path $\beta$ such that $w(\beta)$ is the empty word. This means that $\beta$ is a path in $G^{\prime}$ and, since $G^{\prime}$ is simply connected, $\beta$ is homotopic in $G^{\prime}$ to a trivial path. Therefore, $c$ is $\mathcal{L}$-homotopic in $G$ to the trivial path $(B, B)$.

\section{Fundamental Groups in 3D}

The $3 \mathrm{D}$ case is certainly the most important concerning applications in pattern recognition of algebraic invariants computation. As we shall see, the 3D digital fundamental group, as considered for example in [4], [5], [2] and [1], can be defined as a particular case of graphs fundamental groups as defined in Section 1.3. In this section, after having recalled basic notions and notations of 3D digital topology, we explain how to reduce the number of generators and relations obtained for the presentation of the $3 \mathrm{D}$ fundamental group given by Theorem 2 , first by considering subgraphs of the classical adjacency graphs which have the same fundamental group, and then by simplifying the sets of generators and relations we obtain.

\subsection{Basic Notions of 3D Digital Topology}

Let $x=(i, j, k) \in \mathbb{Z}^{3}$, and $x^{\prime}=\left(i^{\prime}, j^{\prime}, k^{\prime}\right) \in \mathbb{Z}^{3}$.

The points $x$ and $x^{\prime}$ are called $26-$ adjacent if and only if $\max \left(\left|i^{\prime}-i\right|,\left|j^{\prime}-j\right|,\left|k^{\prime}-k\right|\right)=1$. The points $x$ and $x^{\prime}$ are said to be 6 -adjacent if and only if $\left|i^{\prime}-i\right|+\left|j^{\prime}-j\right|+\left|k^{\prime}-k\right|=1$, i.e. if they are 26-adjacent and have two of their coordinates in common. The points $x$ and $x^{\prime}$ are called 18-adjacent if and only if they are 26-adjacent and have at least one of their coordinates in common. Let $n \in\{6,18,26\}$, and $X \subset \mathbb{Z}^{3}$. Given $x \in \mathbb{Z}^{3}$, we denote by $N_{n}(x)$ the set of all $y \in \mathbb{Z}^{3}$ which are $n$-adjacent to $x$. 
Let $X \subset \mathbb{Z}^{3}$ be a subset of $\mathbb{Z}^{3}$. We denote by $G_{n}(X)$ the graph whose vertices are elements of $X$, two vertices of $G_{n}(X)$ being adjacent in $G_{n}(X)$ if and only if they are $n$-adjacent (as points of $\left.\mathbb{Z}^{3}\right)$.

An $n$-path in $X$ is a path in the graph $G_{n}(X)$. An $n$-path $c$ is called closed if, as a path of $G_{n}(X)$, the path $c$ is a loop. A closed path $c=\left(x_{0}, \ldots, x_{p}\right)$ is called simple closed if for $i, j \in\{0, \ldots, p\}$ with $i \neq j(\bmod p)$ we have $x_{i} \neq x_{j}$. The set $X$ is called $n$-connected iff $G_{n}(X)$ is connected, and $n$-connected components of $X$ are defined as connected components of $G_{n}(X)$.

For $X \subset \mathbb{Z}^{3}$ we denote by $\bar{X}$ the complement $\mathbb{Z}^{3} \backslash X$ of $X$. As usual in digital topology ([6]), when we analyze an object with an adjacency relation $n \in\{6,18,26\}$, we have to consider another $\bar{n}$ adjacency relation for $\bar{X}$. We introduce here a difference between the 6 -adjacency relation when associated with the 26-adjacency relation, and when associated with the 18-adjacency relation. We must also introduce a distinct notation for these two things since several notions, as far as the fundamental group is concerned, will be different for these two adjacencies. We denote by $6^{+}$the 6 -adjacency relation when associated with the 18 -adjacency relation. Being $6^{+}$-adjacent, $6^{+}$-connected is just being 6 -adjacent, 6 -connected but, as we already said, several definitions will be different for the 6 and the $6^{+}$ notions.

\subsection{The Digital Fundamental Group}

In this section, we introduce the (digital) $n$-fundamental group of an object $X$. This group, which has been introduced in digital topology by T. Y. Kong ([4] and [5]), represents intuitively the "holes" in $X$. A hole is detected when some closed $n$-path in $X$ can not be deformed to a single point. For instance, in a hollow torus there are two "independant" such closed $n$-paths, and the fundamental group of a hollow torus is, as a group, isomorphic to $\left(\mathbb{Z}^{2},+\right)$. The notion of the fundamental group can be formalized as follows:

Let $X \subset \mathbb{Z}^{3}$. Let us consider a fixed point $B \in X$ called the base point. We denote by $A_{B}^{n}(X)$ the set of all closed $n$-paths $\pi=\left(x_{0}, \ldots, x_{p}\right)$ which are included in $X$ and such that $x_{0}=x_{p}=B$.

To introduce the fundamental group, we first introduce a set $\mathcal{L}_{n}(X)$ of loops, which depends on the considered adjacency relation $n$.

If $n \in\left\{26,18,6^{+}\right\}$, then $\mathcal{L}_{n}(X)$ is the set of all closed $n$-paths of $X$ which are either back and forths, or contained in a $2 \times 2 \times 2$ cube of 8 points.

If $n=6$, then $\mathcal{L}_{n}(X)$ is the set of all closed $n$-paths of $X$ which are either back and forths, or contained in a $2 \times 2$ square of 4 points

Then, two $n$-paths are called $n$-homotopic in $X$ if they are $\mathcal{L}_{n}(X)$-homotopic in the graph $G_{n}(X)$; we denote by $\Pi_{1}^{n}(X, B)$, and we call the $n$-fundamental group of $X$, the $\mathcal{L}_{n}(X)$-fundamental group of $G_{n}(X)$.

It is easily seen that this definition is equivalent to another one which is commonly used and can be described as follows. First we introduce the notion of an elementary n-deformation. Two $n$-paths $\pi$ and $\pi^{\prime}$ in $X$ are said to be the same up to an elementary $n$-deformation (with fixed extremities) if they are of the form $\pi=\pi_{1} * \gamma * \pi_{2}$ and $\pi^{\prime}=\pi_{1} * \gamma^{\prime} * \pi_{2}$, the $n$-paths $\gamma$ and $\gamma^{\prime}$ having the same extremities and being both included in a common $2 \times 2$ square of 4 
points if $n=6$, and in a common $2 \times 2 \times 2$ cube of 8 points if $n=6^{+}, 18$ or 26 .

Now, two $n$-paths $\pi$ and $\pi^{\prime}$ in $X$ are $n$-homotopic in $X$ if there exists a finite sequence of $n$-paths $\pi=\pi_{0}, \ldots, \pi_{m}=\pi^{\prime}$ in $X$ such that for $i=1, \ldots, m$ the $n$-paths $\pi_{i-1}$ and $\pi_{i}$ are the same up to an elementary deformation (with fixed extremities).

The $n$-homotopy relation defines an equivalence relation on $A_{B}^{n}(X)$, which coincides with the $\mathcal{L}_{n}(X)$-homotopy relation, and therefore $\Pi_{1}^{n}(X, B)$ is the set of equivalence classes of this equivalence relation.

Clearly, since the 3D $n$-fundamental group of $X$ has been characterized as the $\mathcal{L}$-fundamental group for a given set $\mathcal{L}$ of loops in a graph (namely the graph $G_{n}(X)$ ), then Theorem 2 provides a way to compute a presentation for $\Pi_{1}^{n}(X)$. However, the number of generators and relations directly obtained by this method is quite huge. In the sequel of this paper, we provide some ways to reduce the size of the presentation of the fundamental group in 3D.

\section{Computing 3D Fundamental Groups}

In the sequel of this paper, $n$ is an adjacency relation in $\left\{6,6^{+}, 18,26\right\}$, and $X$ denotes an $n$-connected subset of $\mathbb{Z}^{3}$.

\subsection{Finite Presentation}

Definition 2 Let $x$ and $y$ be two elements of $X$.

1. The two points $x$ and $y$ are called $d_{6}$-adjacent or $d_{6^{+}}$-adjacent in $X$ if they are 6 -adjacent;

2. The two points $x$ and $y$ are called $d_{18}$-adjacent in $X$ if either they are $d_{6}$-adjacent, or they are 18-adjacent and have no common 6-neighbor in $X$;

3. The two points $x$ and $y$ are called $d_{26}$-adjacent in $X$ if either they are $d_{18}$-adjacent, or they are 26-adjacent and have no common 18-neighbor in $X$.

From the notion of $d_{n}$-adjacency defined above, we can define a graph $D_{n}(X)$ having $X$ as set of vertices, two elements of $X$ being adjacent in $D_{n}(X)$ if and only if they are $d_{n}$-adjacent. Then we define from the graph $D_{n}(X)$ the notion of a $d_{n}$-path in $X$, and a $d_{n}$-connected component in $X$ as we have defined an $n$-path and an $n$-connected component from $G_{n}(X)$.

Lemma 3 Let $c$ be an $n$-path in $X$. Then $c$ is $n$-homotopic to a $d_{n}$-path $c^{\prime}$ in $X$.

Proof: If $n=6$ or $n=6^{+}$, then obviously we can take $c^{\prime}=c$. Now suppose $n=18$. By an elementary $n$-deformation, we can add to $c$ a common 6 -neighbor between two successive points of $c$ which are not $d_{18}$-adjacent in $X$. By doing this for all couples of successive points of $c$ which are not $d_{18}$-adjacent, we obtained the desired $d_{18}-$ path $c^{\prime}$. Finally, suppose $n=26$. By an elementary $n$-deformation, we can add to $c$ a common 18-neighbor between two successive points of $c$ which are not $d_{26}$-adjacent and not 18 -adjacent in $X$. By doing this for all couples of successive points of $c$ which are not $d_{26}$-adjacent and not 18-adjacent, we obtain a 26-path in which any two successive points which are not 18 -adjacent are $d_{26}$-adjacent. Then we can 
add to this 26-path a common 6-neighbor between two successive points of $c$ which are 18-adjacent and not $d_{18}$-adjacent in $X$. By doing this for all couples of successive points which are not $d_{18}$-adjacent, we obtained the desired $d_{26}-$ path $c^{\prime}$.

Remark 1 Let $Y \subset \mathbb{Z}^{3}$. Then, due to Lemma 3, the $d_{n}$-connected components of $Y$ coincide with the $n$-connected components of $Y$.

Definition $3 A d_{n}$-path $c^{\prime}$ constructed from an $n$-path $c$ as in the proof of Lemma 3 is called $d_{n}$-path of $X$ associated to $c$. Note that a $d_{n}$-path of $X$ associated to $c$ is not necessarily unique because some choices are made during its construction.

Now, in order to define the $d_{n}$-fundamental group of $X$, we must define a set $\mathcal{D}_{n}(X)$ of loops in the graph $D_{n}(X)$, as the elements of $\mathcal{L}_{n}(X)$ which are $d_{n}$-paths.

Definition 4 Let $c_{1}$ and $c_{2}$ be two $d_{n}$-paths in $X$. We say that $c_{1}$ and $c_{2}$ are $d_{n}$-homotopic (with fixed extremities) iff they are $\mathcal{D}_{n}(X)$-homotopic in $D_{n}(X)$. Equivalently, the $d_{n}$-paths $c_{1}$ and $c_{2}$ are $d_{n}$-homotopic iff there exists a sequence $\left(\alpha_{1}, \ldots, \alpha_{q}\right)$ of $d_{n}$-paths of $X$, with $\alpha_{1}=c_{1}$ and $\alpha_{q}=c_{2}$, such that for $i=1, \ldots, q-1$ the $d_{n}-$ paths $\alpha_{i}$ and $\alpha_{i+1}$ are, as $n$-paths, the same up to an elementary $n$-deformation.

Now we want to prove that the $\mathcal{D}_{n}(X)$-fundamental group of $D_{n}(X)$ is isomorphic to the $n$-fundamental group of $X$, which shows that we can work on the fundamental group by considering only $d_{n}$-paths. First we should prove one lemma and one remark.

Lemma 4 Let $X \subset \mathbb{Z}^{3}$ and let $c_{1}$, $c_{2}$ be two $n$-paths which are $n$-homotopic. Let $c_{1}^{\prime}$ and $c_{2}^{\prime}$ be $d_{n}$-paths of $X$ respectively associated to $c_{1}$ and $c_{2}$. Then the two $d_{n}$-paths $c_{1}^{\prime}$ and $c_{2}^{\prime}$ are $d_{n}$-homotopic.

Proof: We distinguish three cases:

First case : If $c_{1}=c_{2}$ (only $c_{1}^{\prime}$ differs from $c_{2}^{\prime}$ ). Between two successive points $x_{i}$ and $x_{i+1}$ of $c_{1}$, possibly some points have been inserted to obtain $c_{1}^{\prime}$, and similarly for $c_{2}$. All these points lie in a $2 \times 2$ square if $x_{i}$ is 18 -adjacent to $x_{i+1}$, and in a $2 \times 2 \times 2$ cube otherwise. The result then follows directly from the definition of an elementary $n$-deformation.

Second case: If $c_{1}$ and $c_{2}$ are the same up to an elementary $n$-deformation. Let $S$ be a $2 \times 2$ square if $n=6$, and a $2 \times 2 \times 2$ cube if $n \in\left\{6^{+}, 18,26\right\}$, and let $c_{1}=\gamma_{1} * \gamma * \gamma_{2}$ and $c_{2}=\gamma_{1} * \gamma^{\prime} * \gamma_{2}$, the two $n$-paths $\gamma$ and $\gamma^{\prime}$ having the same extremities an being both contained in $S$. We observe that all the points of any $d_{n}-$ path associated to $\gamma$ or $\gamma^{\prime}$ is contained in $S$. Therefore, any $d_{n}$-path associated to $\gamma$ is the same up to an elementary $n$-deformation as any $d_{n}$-path associated to $\gamma^{\prime}$. The end of the proof in the second case follows from the first case applied to the $n$-paths $\gamma_{1}$, and then to the $n$-path $\gamma_{2}$.

Third case: General case. This case follows directly from the two first cases and the definition of $n$-homotopy between $c_{1}$ and $c_{2}$.

Remark 2 Let $c_{1}$ and $c_{2}$ be two $n$-paths such that the $d_{n}$-paths associated to $c_{1}$ and $c_{2}$ are $d_{n}$-homotopic in $X$. Then $c_{1}$ and $c_{2}$ are $n$-homotopic in $X$. 
Remark 2 follows directly from the definition of the $d_{n}$-paths associated to $c_{1}$ and $c_{2}$, and from the fact that being $d_{n}$-homotopic is a particular case of being $n$-homotopic.

Theorem 3 The $\mathcal{D}_{n}(X)$-fundamental group $\Pi_{1}^{\mathcal{D}_{n}(X)}\left(D_{n}(X), B\right)$ of $D_{n}(X)$ is isomorphic to the $n$-fundamental group $\Pi_{1}^{n}(X, B)$ of $X$.

Proof: Let us consider the map $f$ which to an $n$-path $c$ of $X$ associates a $d_{n}$-paths $f(c)$ associated with $c$. Then, Lemma 4 implies that $f$ induces a map $\tilde{f}: \Pi_{1}^{n}(X, B) \longmapsto$ $\Pi_{1}^{\mathcal{D}_{n}(X)}\left(D_{n}(X), B\right)$. Clearly, the map $f$ preserves the concatenation of paths, and $\widetilde{f}$ is therefore a group morphism. Since for any $d_{n}$-path $c$ we have $f(c)=c$, the map $f$, and therefore the map $\tilde{f}$ is onto. Now, from Remark 2, the map $\tilde{f}$ is one to one. Hence, the map $\tilde{f}$ is a group isomorphism.

From Theorem 3 follows that, in order to compute a presentation of the $n$-fundamental group of $X$, we can equivalently compute a presentation of the $\mathcal{D}_{n}(X)$-fundamental group of $D_{n}(X)$. If we compute the presentations using the method described in Section 2, the number of generators of the obtained presentation is much lower if we consider the graph $D_{n}(X)$ rather than the $n$-adjacency graph. Indeed, the number of edges of a covering tree is equal to $\operatorname{card}(X)-1$ for both graphs, so that the number of generators obtained is the number of edges of the graph minus card $(X)$ plus 1 . Now, the number of edges of $D_{n}(X)$ is generally lower than the number of edges of the $n$-adjacency graph. For instance, a point $x \in X$ such that $N_{26}(x) \subset X$ ( $x$ is an interior point) has $6 d_{26}$-neighbors and 2626 -neighbors. Now, in order to reduce the number of relators of the obtained presentation, we show that the set of null loops used to define the $\mathcal{D}_{n}(X)$-fundamental group can be reduced to simple closed $d_{n}$-paths of $\mathcal{D}_{n}(X)$.

Lemma 5 Let $c$ be a closed $d_{n}-$ path in $X$. Then $c$ can be obtained from a $d_{n}-$ path $(x, x)$ (reduced to a single point) by inserting iteratively simple closed $d_{n}-$ paths.

Proof of Lemma 5: Let $c=\left(x_{0}, \ldots, x_{p}\right)$. If $c$ is simple, there is nothing to prove. Otherwise, let $i$ be the smallest integer such that there exists $j \neq i$ with $x_{i}=x_{j}$, and let $j$ be the smallest integer distinct from $i$ such that $x_{i}=x_{j}$.

The closed $d_{n}$-path $s=\left(x_{i}, \ldots, x_{j}\right)$ is simple, and $c$ is obtained from the $d_{n}$-path $c_{1}=$ $\left(x_{0}, \ldots, x_{i}, x_{j+1}, \ldots, x_{p}\right)$ by inserting the simple closed $d_{n}$-path $s$.

If $c_{1}$ is simple closed, there is nothing else to prove. Otherwise, we construct a closed $d_{n}$-path $c_{2}$ from $c_{1}$ as we have constructed $c_{1}$ from $c$, and we iterate the process. Since the length of the $d_{n}$-paths decreases at each step, we obtain by this process a simple closed $d_{n}$ - path $c_{i}$.

Now let $\mathcal{M}_{n}(X)$ be the set of loops of $\mathcal{D}_{n}(X)$ which are simple closed.

Lemma 6 Let $c_{1}$ and $c_{2}$ be two closed $d_{n}$-paths. Then $c_{1}$ and $c_{2}$ are $d_{n}$-homotopic in $X$ if and only if they are $\mathcal{M}_{n}(X)$-homotopic in $D_{n}(X)$. 
Proof: Clearly, since $\mathcal{M}_{n}(X) \subset \mathcal{D}_{n}(X)$, if $c_{1}$ and $c_{2}$ are $\mathcal{M}_{n}(X)$-homotopic in $D_{n}(X)$, they are also $d_{n}$-homotopic in $X$. Conversely, we can assume without loss of generality that $c_{1}=\pi_{1} * \gamma * \pi_{2}$ and $c_{2}=\pi_{1} * \gamma^{\prime} * \pi_{2}$, the $d_{n}$-path $\gamma * \gamma^{\prime-1}$ being a loop of $\mathcal{D}_{n}(X)$. Then $c_{1}$ is $\mathcal{M}_{n}(X)$-homotopic to the $d_{n}$-path $c_{1}^{\prime}=\pi_{1} * \gamma * \gamma^{\prime-1} * \gamma^{\prime} * \pi_{2}$. Now, from Lemma 5 and the definition of $\mathcal{M}_{n}$, the $d_{n}$-path $\gamma * \gamma^{\prime-1}$ is $\mathcal{M}_{n}(X)$-homotopic to a trivial path reduced to a single point. Therefore, the path $c_{1}^{\prime}$ is in turn $\mathcal{M}_{n}(X)$-homotopic to the $d_{n}$-path $\pi_{1} * \gamma^{\prime} * \pi_{2}=c_{2}$

Corollary 1 The $\mathcal{M}_{n}$-fundamental group of $D_{n}(X)$ is equal to the $\mathcal{D}_{n}(X)$-fundamental group of $D_{n}(X)$.

Therefore, in order to compute a presentation of the $n$-fundamental group of $X$, we can compute a presentation of the $\mathcal{M}_{n}$-fundamental group of $D_{n}(X)$. So, the number of relators we have to consider in the presentation is the cardinality of $\mathcal{M}_{n}(X)$, which is the number of simple closed $d_{n}$-paths in $X$ which are contained in a $2 \times 2 \times 2$ cube of 8 points if $n \in\left\{6^{+}, 18,26\right\}$, and contained in a $2 \times 2$ square of 4 points if $n=6$. In particular, the presentation obtained in so doing is finite.

\subsection{Reducing the Number of Generators and Relators}

First we explain how to reduce the number of generators. The idea is simple: if one of the relators is of the form $a_{i}^{\varepsilon_{1}} a_{j}^{\varepsilon_{2}}$, with $i, j \in\{1, \ldots, m\}$, with $i \neq j$ and $\varepsilon_{i}, \varepsilon_{j} \in\{-1,+1\}$, then we can express the generator $a_{j}$ using the generator $a_{i}$, so that the system obtained by removing the generator $a_{j}$ is still a system of generators. We then simply change each occurrence of $a_{j}$ by its expression using $a_{i}$ in each relator. Note that the same principle applies if one of the relators contains exactly one occurrence of a symbol $a_{i}$, but this increases the length of the relators.

Now we explain how to reduce the number of relators. Let us first recall that the set $\mathcal{R}$ of all defined relators is the set of all words $w(\alpha)$, where $\alpha$ is any simple closed $d_{n}$-path contained in $X \cap S$, where $S$ is any $2 \times 2$ square if $n=6$, and any $2 \times 2 \times 2$ cube if $n \in\left\{26,18,6^{+}\right\}$.

Now, for a fixed $S$, the closed $d_{n}$-paths contained in $X \cap S$ are not all independent. First, if a closed $d_{n}$-path $\alpha$ is a cyclic permutation of another $d_{n}$-path $\beta$, then so are the words $w(\alpha)$ and $w(\beta)$. Since words being the same up to a cyclic permutation of symbols correspond to conjugate elements of $\mathcal{F}_{m}$, then removing, say, $\beta$ from the set $\mathcal{R}$ does not affect the generated normal subgroup $H$, and therefore the group $\Gamma$. Finally, note that if one closed $d_{n}-$ path $\alpha$ in $X \cap S$ is obtained form the concatenation of two or more closed $d_{n}$-paths of $D \cap S$ by removing points which have their predecessor and their successor equal, then the relator $w(\alpha)$ is a consequence of all other relators and therefore it can be removed from $\mathcal{R}$. Note that the set of the relators to be considered for $X \cap S$ depends only of the set $X \cap S$, and can be computed once for all for all of the $2^{8}$ subsets of a $2 \times 2 \times 2$ cube. 


\section{Conclusion}

We can compute a presentation for the fundamental group of an arbitrary graph with an arbitrary set of null loops, and a finite presentation of the fundamental group of any subset of $\mathbb{Z}^{3}$, for any chosen adjacency relation $n \in\left\{26,18,6,6^{+}\right\}$. The presentation obtained in $3 D$ satisfies some special properties (for example, the length of all the relators is less than or equal to 8$)$.

Some computation problems in groups such as the word problem (determining if a given word is equal to 1 in a group $\Gamma$ given by generators and relations), and the isomorphism problem (determining if two groups given by generators and relations are isomorphic) are not decidable in the general case. It would be interesting to see if these problems are decidable for the particular presentations of groups we obtain in 3D, due to their specific properties. Indeed, a solution to these problems could enable us to decide if a given closed path can be continuously deformed into another path, and if two given objects have isomorphic fundamental groups, which is a central question of pattern recognition.

\section{References}

[1] G. Bertrand, Simple points, topological numbers and geodesic neighbourhoods in cubic grids, Pattern Rec. Letters, 15, pp 1003-1011, 1994.

[2] S. Fourey and R. Malgouyres, Linking Numbers and a New Characterization of 3D Simple Points, Submitted for publication.

[3] S. Fourey and R. Malgouyres, Intersection Number and Topology Preservation Within Digita Surfaces, Proceedings of the sixth International Workshop on Parallel Image Processing and Analysis (IWPIPA'99), Madras, India, pp 138-158, January 1999.

[4] T. Y. Kong, A Digital Fundamental Group Computer and Graphics, 13, pp 159-166, 1989.

[5] T. Y. Kong, Polyhedral Analogs of Locally Finite Topological Spaces R. M. Shortt editor, General Topology and Applications: Proceedings of the 1988 Northeast Conference, Middletown, CT (USA), Lecture Notes in Pure and Applied Mathematics, 123, pp 153164, 1990.

[6] T. Y. Kong and A. Rosenfeld, Digital Topology : Introduction and Survey Comp. Vision, Graphics and Image Processing, 48, pp 357-393, 1989.

[7] A. Lenoir, Fast Estimation of Mean Curvature on the Surface of a 3D Discrete Object, Proceeding of DGCI'97, Lecture Notes in Computer Science 1347, pp 175-186, 1997.

[8] R. Malgouyres, Homotopy in 2-dimensional Digital Images, Theoretical Computer Science, vol. 230, pp 221-233, 2000.

[9] R. Malgouyres, Presentation of the Fundamental Group in Digital Surfaces, Proceedings, of DGCI'99, Lecture Notes in Computer Science 1568, pp 136-150, 1999. 
[10] R. Malgouyres, A. Lenoir, Topology Preservation Within Digital Surfaces, Graphical Models, 62, pp 71-84 (2000). 